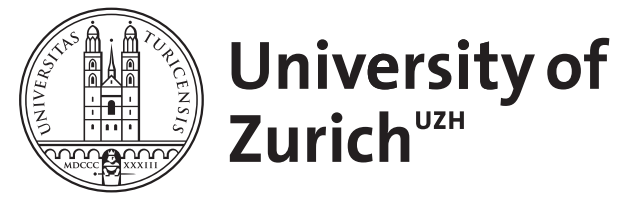

Zurich Open Repository and Archive

University of Zurich

University Library

Strickhofstrasse 39

CH-8057 Zurich

www.zora.uzh.ch

Year: 2017

\title{
Management of Elderly Patients with Glioblastoma
}

Roth, Patrick ; Gramatzki, Dorothee ; Weller, Michael

DOI: https://doi.org/10.1007/s11910-017-0740-3

Posted at the Zurich Open Repository and Archive, University of Zurich

ZORA URL: https://doi.org/10.5167/uzh-141058

Journal Article

Accepted Version

Originally published at:

Roth, Patrick; Gramatzki, Dorothee; Weller, Michael (2017). Management of Elderly Patients with Glioblastoma. Current Neurology and Neuroscience Reports:17:35.

DOI: https://doi.org/10.1007/s11910-017-0740-3 
Management of elderly patients with glioblastoma

Patrick Roth $^{1 *}$, Dorothee Gramatzki ${ }^{1}$, Michael Weller $^{1}$

${ }^{\mathbf{1}}$ Department of Neurology and Brain Tumor Center, University Hospital Zurich and University of Zurich, Switzerland

*Correspondence: Dr. Patrick Roth, Department of Neurology, University Hospital

Zurich, Frauenklinikstrasse 26, 8091 Zurich, Switzerland, Tel.: +41 (0)44 255 5511, Fax: +41 (0)44 255 4380, E-mail: patrick.roth@usz.ch

Short title: Glioblastoma in the elderly

Keywords: glioblastoma, elderly, MGMT, temozolomide, radiotherapy 


\begin{abstract}
Purpose of review: Glioblastoma represents one of the major challenges in neurooncology and approximately half of the patients are 60 years or older. We summarize the particular situation of elderly glioblastoma patients with a focus on therapeutic considerations.
\end{abstract}

Recent findings: Favorable molecular markers such as mutations in the isocitrate dehydrogenase (IDH) 1 or 2 genes are virtually absent in glioblastomas in elderly patients. Treatment options are similar to the situation in young patients and comprise surgical resection, radiation therapy and alkylating chemotherapy. The performance status as well as comorbidities may have a stronger impact on the individual treatment decision than in young patients. The MGMT promotor methylation status allows for a stratification of treatment beyond the surgical intervention. In patients with MGMT promoter methylated tumors, monotherapy with temozolomide is superior to radiotherapy alone. Similarly, the benefit conferred by combined temozolomide-based chemoradiotherapy compared to radiotherapy alone is largely restricted to patients with MGMT promoter methylated tumors. At recurrence, no standard treatment has been established.

Summary: The prognosis for elderly patients with glioblastoma remains poor. The MGMT promoter methylation status helps guiding treatment decisions and withholding inactive, but potentially harmful treatments. 


\section{Introduction}

Glioblastoma remains one of the major challenges in neurooncology. In contrast to the consistently increasing knowledge on the underlying biology of these tumors, only little progress has been made in the clinical setting. Glioblastoma is a tumor thought to be of neuroglial origin, typically characterized by astrocytic differentiation and additional histological features such as microvascular proliferation and necrosis (1). On the molecular level, no major differences have been found in glioblastomas from young and elderly patients except for the frequency of mutations in the isocitrate dehydrogenase (IDH) 1 and 2 genes. IDH mutations occur in approximately $5 \%$ of all glioblastomas and are associated with an improved prognosis (2). They are virtually absent in glioblastomas of elderly patients. Yet, the updated 2016 WHO classification indicates that IDH-mutantglioblastoma should be considered a distinct entity. Glioblastomas are diagnosed throughout all age groups with a median age at diagnosis of approximately 64 years (3). In patients aged 65 years or older, glioblastoma accounts for the vast majority of all gliomas and is much more frequent than WHO grade II or III gliomas. Hence, a significant proportion of patients is at an advanced age when the tumor is diagnosed. According to age-adjusted analyses, the incidence of glioblastoma is continuously increasing from young to old subjects with a highest incidence rate in patients aged 75 to 84 years (3). Is has remained unclear why these tumors become more frequent in the elderly. Host factors such as a less efficient function of the immune system may be a contributing factor but solid data which support this hypothesis are largely lacking. The dataset published by the Central Brain Tumor Registry of the United States (CBTRUS) indicates a 1-year survival rate for glioblastoma patients aged 65-74 years of $28.7 \%$ and for patients aged 75 years or older of only $12.1 \%$. Five years after the diagnosis, only $2.4 \%$ of the 
patients aged 65 to 74 and $1.1 \%$ of the patients aged 75 or older are alive (3).

In most countries around the world, the life expectancy is continuously raising. As a result, it must be assumed that the number of elderly patients affected by glioblastoma will increase within the next years and decades. Hence, there is a considerable need of improved therapeutic strategies for elderly glioblastoma patients. In sharp contrast, only few randomized trials have been specifically designed and performed for this patient population. In many other trials where age was not a specific exclusion criterion, elderly patients are typically underrepresented because of frailty, comorbidities, the need of medication which is not compatible with study procedures or other reasons. Furthermore, there are frequently concerns regarding a potential quality of life impairment due to side effects of study drugs or other procedures which ultimately preclude enrolment in clinical trials. Thus, compared to younger patients, physicians and caregivers can only partially rely on robust data when it comes to treatment decisions. In the following, we summarize the current knowledge on treatment options for elderly glioblastoma patients.

\section{Biopsy or resection?}

Despite the characteristic features of glioblastoma that are found by computed tomography $(\mathrm{CT})$ or magnetic resonance imaging (MRI), tissue is required for a definite diagnosis and increasingly also molecular marker assessment. Therefore, a biopsy or surgical resection is mandatory to allow for a histopathological characterization of the tumor tissue. The emergence of various molecular markers stresses the importance of a sufficient amount of tissue in order to be able to perform all desired analyses.

It has long been discussed whether a gross total resection results in prolonged survival 
in glioblastoma patients. A small prospective trial addressed this question in elderly patients with suspected glioblastoma. Patients older than 65 years were eligible and 30 patients were randomized to undergo stereotactic biopsy or open craniotomy and resection. Only 19 patients were finally diagnosed with glioblastoma, 4 additional patients had an anaplastic glioma. These patients were subsequently treated with radiotherapy. The median overall survival (OS) was 171 days after craniotomy compared to 85 days after biopsy $(\mathrm{p}=0.035)$. Despite its obvious limitations such as the small sample size as well as the restriction of further treatment to radiotherapy, these data suggested that tumor resection is beneficial and prolongs the survival of elderly glioblastoma patients (4). These data are further supported by a number of retrospective analyses of larger patient cohorts. A case-control study compared 40 glioblastoma patients aged 65 years or older to 40 patients matched for age, performance status, localization of the tumor and treatment beyond surgery. Tumor resection was associated with a longer median OS than biopsy (5.7 vs 4.0 months, $\mathrm{p}=0.02$ ) (5). Another retrospective series of 142 elderly patients with newly diagnosed glioblastoma reported a median OS of 13.0 months following resection compared to only 4.0 months after biopsy $(\mathrm{p}<0.001)(6)$. Multivariate analyses of the randomized phase III studies, NOA-08 and Nordic (see below for details), also indicated that surgical resection is superior to biopsy alone $(7,8)$. Finally, there are data from prospective trials which took advantage of the fluorescent dye, 5-aminolevulinic acid (5-ALA), or intraoperative MRI in order to improve the extent of resection $(9,10)$. Both trials demonstrate that gross total resection is associated with prolonged progression-free survival (PFS). These trials enrolled mainly younger glioblastoma patients and the extrapolation to the elderly population must be done with caution. However, in light of all available data, it can be concluded that tumor resection 
contributes to prolonged survival compared to biopsy also in elderly glioblastoma patients.

\section{The role of radiotherapy}

Because of the overall poor prognosis of elderly glioblastoma patients, it must be carefully assessed whether any kind of tumor-specific treatment beyond surgery is warranted. This is of particular importance in frail patients with comorbidities who may be unable to tolerate radiotherapy or pharmacotherapy or both and suffer from side effects with consequent impairment of their quality of life. Furthermore, there have been speculations that the benefit of any treatment beyond surgery regarding survival times would be minimal in the elderly. This question was addressed in a randomized trial in patients with newly diagnosed glioblastoma aged 70 or older. Following histological confirmation of the diagnosis, 85 patients were randomized to receive either best supportive care alone or postsurgical radiotherapy with 50 Gy in fractions of $1.8 \mathrm{~Gy}$ fractions in addition to supportive care. The combination of radiotherapy and supportive care resulted in a prolongation of progression-free survival (14.9 weeks vs. 5.4 weeks). The median overall survival, which was the primary endpoint, was 29.1 weeks for patients treated with radiotherapy plus supportive care but only 16.9 weeks in the cohort of patients receiving supportive care alone (HR 0.47; $\mathrm{p}=0.002$ ). Regarding toxicity, radiotherapy was well tolerated and no severe adverse events related to radiotherapy were reported. Furthermore, there were no significant differences between the 2 cohorts in the evaluation of quality-of-life and cognitive function (11). It can therefore be concluded that radiotherapy is safe and active in elderly glioblastoma patients.

One of the drawbacks of radiotherapy is the fact that the patients need to go the 
hospital on a daily base for several weeks. Data from several trials support the use of hypofractionated radiotherapy in elderly patients. A trial comparing $40 \mathrm{~Gy}$ in 15 fractions with standard radiotherapy consisting of $60 \mathrm{~Gy}$ in 30 fractions did not show a difference in overall survival which was 5.6 months for the shorter course and 5.1 months for the standard schedule (12). Because of this study as well as data from other trials (e.g., Nordic, see below for details), hypofractionated radiotherapy has become a standard of care for elderly glioblastoma patients.

In a trial performed by the International Atomic Energy Agency, hypofractionated radiotherapy with 40 Gy in 15 fractions was compared to a very short course radiotherapy consisting of $5 \times 5$ Gy. Patients with newly diagnosed glioblastoma aged 65 years or older and patients aged 50 years or older with a Karnofsky performance score (KPS) between 50 and 70\% were eligible. Between 2010 and 2013, 98 patients were enrolled. There was no difference regarding overall survival between the 2 groups with a median OS of 7.9 months in the $5 \times 5$ Gy cohort and 6.4 months in the group of patients receiving 40 Gy in 15 fractions ( $\mathrm{p}=0.988$ ). The median PFS was 4.2 months in both groups. No difference in quality of life was noticed at 4 and 8 weeks after treatment (13). However, because of the limited follow-up time of only 6.4 months, a potential negative impact of the short one-week radiotherapy on the cognitive function of patients surviving for more than few months cannot be excluded. Thus, it remains doubtful that this radiation schedule will be embraced in countries where molecular testing and individualized treatment approaches are becoming increasingly available.

\section{Alkylating chemotherapy}

Since the introduction of temozolomide to the standard of care for younger 
glioblastoma patients, it has gained widespread use (14). It is available as an oral formulation and overall well tolerated which makes it convenient for patients as it does not require daily treatment in a hospital. However, its use in elderly glioblastoma patients was not established for many years because of lacking data from prospective studies. Monotherapy with temozolomide was assessed in a non-randomized phase II trial in glioblastoma patients aged 70 or older and a postoperative KPS of less than $70 \%$. The drug was administered at a dose of $150-200 \mathrm{mg} / \mathrm{m}^{2}$ for 5 days every 28 days. Temozolomide was overall well tolerated with grade 3 to 4 neutropenia and thrombocytopenia in $13 \%$ and $14 \%$ of patients, respectively. Median PFS was 16 weeks and median OS was 25 weeks. In 23 out of 70 enrolled patients, KPS improvement of at least 10 points was noticed. Furthermore, an improvement of quality of life and cognitive function was reported. As expected, a methylated $\mathrm{O}^{6}$ methylguanine-DNA methyltransferase (MGMT) promoter in the tumor tissue was associated with longer PFS and OS (15). Although a control arm of patients who received only best supportive care was lacking, the authors concluded that temozolomide is active as single agent treatment.

\section{Trials comparing radiotherapy and chemotherapy}

The therapeutic activity of monotherapy with temozolomide compared to radiotherapy in elderly patients with newly diagnosed glioblastoma was addressed in 2 randomized phase III studies. The Nordic trial randomly assigned 291 elderly patients aged 60 years or older with newly diagnosed glioblastoma to one of three different treatment regimens: i) monotherapy with temozolomide given at 5 out of 28 days with $150-200 \mathrm{mg} / \mathrm{m}^{2}$, ii) standard radiotherapy with 60 Gy in 30 fractions or iii) hypofractionated radiotherapy of $10 \times 3.4=34 \mathrm{~Gy}$ (8). In the intention to treat (ITT) 
population, there was no difference in the outcome of patients treated with temozolomide or hypofractionated radiotherapy (HR 0.82). Somewhat surprisingly, patients who were treated with hypofractionated radiotherapy had a significantly longer median OS than patients who received standard radiotherapy within the ITT population in the subgroup of patients aged 70 years or older ( 7.0 vs 5.2 months, $\mathrm{p}=0.02$ ). This might be explained by the fact that more patients in the standard radiotherapy cohort did not complete the radiotherapy schedule. These data further support the use of hypofractionated radiotherapy in elderly glioblastoma patients as indicated above.

A similar study, the randomized phase III NOA-08 trial, assessed monotherapy with temozolomide administered in a dose-intensified regimen of $100 \mathrm{mg} / \mathrm{m}^{2}$ given on days 1-7 every other week and compared this regimen to standard radiotherapy consisting of 60 Gy given in 30 fractions. Patients aged 65 years or older with newly diagnosed glioblastoma (89\%) or anaplastic astrocytoma (11\%) were enrolled. Regarding overall survival, temozolomide was non-inferior to radiotherapy (HR 1.09). The overall survival rate after 12 months was $34.4 \%$ in the cohort of patients treated with temozolomide and $37.4 \%$ in the radiotherapy group (7). Most importantly, the results of the Nordic and the NOA-08 trials suggest that the survival benefit conferred by temozolomide is largely restricted to patients with tumors harboring a methylated MGMT promoter. MGMT promoter methylation was predictive for benefit from temozolomide treatment whereas patients with MGMT promoter unmethylated tumors displayed a trend for inferior survival. In contrast, the MGMT promoter methylation status was not associated with differential therapeutic activity of radiotherapy. 


\section{Combined radiochemotherapy}

Subgroup analyses of the pivotal EORTC/NCIC trial that resulted in temozolomidebased radiochemotherapy as the new standard of care for younger patients with newly diagnosed glioblastoma had indicated that the benefit conferred by the addition of temozolomide is vanishing with increasing age $(14,16)$. Particularly in patients aged 65 or older, no increase in survival was observed. Of note, the trial was not powered for these subgroup analyses. Still, because of these data as well as the results of the NOA-08 and Nordic trials, most European centers used either radiotherapy alone or monotherapy with temozolomide in elderly glioblastoma patients according to the MGMT promoter methylation status. The addition of temozolomide to radiotherapy compared to radiotherapy alone in patients with newly diagnosed glioblastoma aged 65 years or older was finally examined in the NCIC/EORTC phase III trial (NCT00482677). Radiotherapy was administered with 40 Gy in 15 fractions. In the experimental group, concomitant temozolomide $\left(75 \mathrm{mg} / \mathrm{m}^{2}\right.$ daily during radiotherapy) and maintenance treatment with temozolomide $\left(150-200 \mathrm{mg} / \mathrm{m}^{2}\right.$ on days $1-5$ of a 28 days cycle) for up to 12 cycles or until progression was added. A total of 562 patients were enrolled. As expected, the addition of temozolomide resulted in hematological toxicity that was manageable in most patients. The median OS in patients treated with radiotherapy alone was 7.6 months versus 9.3 months in patients treated with radiochemotherapy $(\mathrm{HR}=0.67 ; \mathrm{p}<0.0001)$. The addition of temozolomide also resulted in a prolonged PFS of 5.3 months versus 3.9 months $(H R=0.50 ; p<0.001)$. Patients with MGMT promoter-methylated tumors had a strong benefit from temozolomide chemotherapy with a median OS of 13.5 months compared to only 7.7 months in the radiotherapy group $(\mathrm{HR}=0.53 ; \mathrm{p}<0.0001)$. In patients with MGMT unmethylated tumors, there was a non-significant trend favoring the 
radiochemotherapy group (median OS 10.0 months vs. 7.9 months; HR=0.75; $\mathrm{p}=0.055$ ) (17). Of note, the results for all subgroups analyses stratified for the MGMT promoter methylation status are better than for the overall cohort suggesting that patients with a poor outcome, most likely patients who had a biopsy only, are underrepresented in these analyses. The publication of the full dataset needs to be awaited to allow for final conclusions.

Based on the results of this study as well as the Nordic and NOA-08 trials, temozolomide-based radiochemotherapy might be considered as a standard of care in elderly glioblastoma patients with a good KPS, allowing for combined modality treatment, and tumors harboring a methylated MGMT promoter. Patients with MGMT promoter unmethylated tumors may still be candidates for radiotherapy alone (Figure 1). Monotherapy with temozolomide might be an option if the MGMT promoter is methylated and combined radiochemotherapy is considered inappropriate because of a reduced KPS or an extensive field of radiotherapy or other reasons.

\section{Beyond radiotherapy and alkylating chemotherapy}

There has been a long-lasting interest in finding additional treatment options for glioblastoma patients. The monoclonal antibody bevacizumab targets vascular endothelial growth factor (VEGF). First reports on its administration to glioblastoma patients were followed by a period of optimism regarding its potential implementation into the standard of care. Initially, the situation seemed to be particular promising for elderly glioblastoma patients. According to a retrospective single center series, treatment with bevacizumab was supposed to have a more pronounced effect in recurrent glioblastoma patients with advanced age (18). It was assumed that this may reflect differences in the biology of tumors in young compared to older patients. 
Similarly, data from early prospective studies pointed to a benefit from bevacizumab treatment in frail or elderly patients $(19,20)$. However, this optimistic view was not endorsed by subgroup analyses from 2 large randomized phase III trials assessing the addition of bevacizumab to temozolomide-based radiochemotherapy in patients with newly diagnosed glioblastoma. Both trials, AVAglio and RTOG 0825, demonstrated that bevacizumab prolongs progression-free survival but has no impact on overall survival $(21,22)$. Of note, none of these trials was designed to specifically clarify the role for bevacizumab in the context of elderly glioblastoma patients. However, there was a significant proportion of patients aged 65 years or older in both studies. There was a trend for more benefit from beacizumab in elderly patients with MGMT promoter unmethylated tumors in the AVAglio trial. Yet, frail patients were underrepresented as KPS was an inclusion criterion. A randomized phase II study assigned elderly glioblastoma patients to hypfractionated radiotherapy alone or radiotherapy plus bevacizumab (ARTE, NCT01443676). The results of this trial will define whether bevacizumab might be further investigated in the context of elderly glioblastoma patients, particularly in those with MGMT promoter-unmethylated tumors. The elderly patient population may be considered particularly suitable to explore novel agents which should be tested initially in combination with radiotherapy or temozolomide alone, but not combined modality treatment. Such an approach is pursued by the EORTC Brain Tumor Group for a the novel multikinase inhibitor TG02 (23).

\section{Treatment at recurrence}

No standard of care has been established so far for elderly glioblastoma patients when the tumor recurs. Obviously, the situation is not much different in younger patients 
where positive data from randomized trials in patients with recurrent glioblastoma are largely lacking. Basically, re-resection can be offered but only a minority of elderly patients are considered appropriate candidates for such an approach due to comorbidities or tumor dimensions which preclude a gross total resection. Depending on the performance status, prior treatment and time between last treatment and tumor recurrence, several options may be available. Radiotherapy should be used when the tumor recurs in elderly patients with tumors harboring a methylated MGMT promoter who had first-line therapy with temozolomide monotherapy $(7,8)$. If not used as firstline treatment, chemotherapy with temozolomide or lomustine/CCNU can be considered. The use of alkylating chemotherapy, however, is of limited benefit in patients with MGMT promoter-unmethylated tumors. Re-exposure to temozolomide is a salvage treatment that might be considered in patients with MGMT promoter methylated tumors following an interval with stable disease after initial treatment with the drug (24).

Novel compounds that have been explored in phase III trials in patients with recurrent glioblastoma comprise enzastaurine and cediranib. Both drugs did not confer a survival benefit compared to lomustine. Furthermore, only a minority of patients were frail or elderly. Bevacizumab has been approved in the US and some other countries in patients with recurrent glioblastoma. However, the combination of bevacizumab and lomustine did not prolong overall survival in patients with recurrent glioblastoma compared to lomustine alone. Subgroup analyses did not point to an activity of bevacizumab in elderly patients (25). In summary, the treatment for elderly patients with progressive or recurrent glioblastoma has not been standardized and randomized trials would be required to make significant progress. 


\section{Current drug development and outlook}

Further research efforts in glioblastoma patients that are currently being investigated involve mainly immunotherapeutic approaches (26). Beyond vaccines which have been explored in neurooncology for several decades, there is a major interest in the use of checkpoint inhibitors. This class of new drugs comprises antibodies which target immune cell receptors or the corresponding ligands in order to strengthen and prolong anti-tumor immune responses (27). Immune checkpoint inhibitors such as the programmed cell death (PD)-1-blocking antibodies nivolumab or pembrolizumab have been approved for various cancers and are currently being tested in clinical trials in patients with newly diagnosed as well as recurrent glioblastoma (28). Although many of the ongoing trial do not specifically exclude elderly patients, it remains even more doubtful than in younger patients whether immunotherapy will result in clinical benefit in the elderly. First, the immune system is supposed to be less active in the elderly and whether a therapeutic intervention results in a clinically meaningful antitumor immune responses is unclear. Second, some immunotherapeutics can exert severe side effects, particularly when different checkpoint inhibitors are used in combination. It must be assumed that elderly patients will again be underrepresented in the ongoing phase II and III trials which explore the activity of vaccines and checkpoint inhibitors (29). Similar to the conventional treatment options, welldesigned trials particularly addressing the needs of older patients will be required to define the role of immunotherapy in the treatment of these patients.

\section{Conclusions}

- Glioblastoma is more frequently diagnosed in the elderly for unknown reasons. 
- Treatment options after biopsy or resection include radiotherapy and chemotherapy with the alkylating agent, temozolomide.

- The Nordic and NOA-08 trials demonstrate a predictive role of MGMT promoter methylation for benefit from temozolomide treatment alone as opposed to radiotherapy alone. Hence, assessment of the MGMT promoter methylation status is warranted in elderly patients in order to define the ideal therapeutic strategy.

- Temozolomide is typically used in the 5/28 days regimen because of the similar results from the Nordic and NOA-08 trials. Furthermore, data from the RTOG 0525 trial which mainly enrolled younger glioblastoma patients, do not point to a superiority of a dose-intensified temozolomide regimen (30).

- Bevacizumab does probably not prolong the survival of elderly glioblastoma patients.

- No standard of care has been established for elderly patients with recurrent glioblastoma.

\section{References}

1. Louis DN, Perry A, Reifenberger G, von Deimling A, Figarella-Branger D, Cavenee WK, et al. The 2016 World Health Organization Classification of Tumors of the Central Nervous System: a summary. Acta Neuropathol. 2016 Jun;131(6):803-20. PubMed PMID: 27157931.

2. Hartmann C, Hentschel B, Wick W, Capper D, Felsberg J, Simon M, et al. Patients with IDH1 wild type anaplastic astrocytomas exhibit worse prognosis than IDH1-mutated glioblastomas, and IDH1 mutation status accounts for the unfavorable 
prognostic effect of higher age: implications for classification of gliomas. Acta Neuropathol. 2010 Dec;120(6):707-18. PubMed PMID: 21088844.

- A comprehensive analyses indicating the strong prognostic role of the IDH mutation status

3. Ostrom QT, Gittleman H, Xu J, Kromer C, Wolinsky Y, Kruchko C, et al. CBTRUS statistical report: primary brain and other central nervous system turmos diagnosed in the United States in 2009-2013. Neuro Oncol. 2016;18 Suppl 5:v1-v75. 4. Vuorinen V, Hinkka S, Farkkila M, Jaaskelainen J. Debulking or biopsy of malignant glioma in elderly people - a randomised study. Acta Neurochir (Wien). 2003 Jan;145(1):5-10. PubMed PMID: 12545256.

5. Chaichana KL, Garzon-Muvdi T, Parker S, Weingart JD, Olivi A, Bennett R, et al. Supratentorial glioblastoma multiforme: the role of surgical resection versus biopsy among older patients. Ann Surg Oncol. 2011 Jan;18(1):239-45. PubMed PMID: 20697823. PMCID: PMC4612568.

6. Oszvald A, Guresir E, Setzer M, Vatter H, Senft C, Seifert V, et al. Glioblastoma therapy in the elderly and the importance of the extent of resection regardless of age. J Neurosurg. 2012 Feb;116(2):357-64. PubMed PMID: 21942727. 7. Wick W, Platten M, Meisner C, Felsberg J, Tabatabai G, Simon M, et al. Temozolomide chemotherapy alone versus radiotherapy alone for malignant astrocytoma in the elderly: the NOA-08 randomised, phase 3 trial. Lancet Oncol. 2012 Jul;13(7):707-15. PubMed PMID: 22578793.

-• The first trial demonstrating that combined radiochemotherapy may be superior to radiotherapy alone in elderly glioblastoma patients

8. Malmstrom A, Gronberg BH, Marosi C, Stupp R, Frappaz D, Schultz H, et al. Temozolomide versus standard 6-week radiotherapy versus hypofractionated 
radiotherapy in patients older than 60 years with glioblastoma: the Nordic randomised, phase 3 trial. Lancet Oncol. 2012 Sep;13(9):916-26. PubMed PMID: 22877848.

- A randomized study comparing 2 different radiation regimens and temozolomide monotherapy in elderly glioblastoma patients

9. Stummer W, Pichlmeier U, Meinel T, Wiestler OD, Zanella F, Reulen HJ. Fluorescence-guided surgery with 5-aminolevulinic acid for resection of malignant glioma: a randomised controlled multicentre phase III trial. Lancet Oncol. 2006 May;7(5):392-401. PubMed PMID: 16648043. Epub 2006/05/02. eng.

10. Senft C, Bink A, Franz K, Vatter H, Gasser T, Seifert V. Intraoperative MRI guidance and extent of resection in glioma surgery: a randomised, controlled trial. Lancet Oncol. 2011 Oct;12(11):997-1003. PubMed PMID: 21868284.

11. Keime-Guibert F, Chinot O, Taillandier L, Cartalat-Carel S, Frenay M, Kantor G, et al. Radiotherapy for glioblastoma in the elderly. N Engl J Med. 2007 Apr 12;356(15):1527-35. PubMed PMID: 17429084.

12. Roa W, Brasher PM, Bauman G, Anthes M, Bruera E, Chan A, et al. Abbreviated course of radiation therapy in older patients with glioblastoma multiforme: a prospective randomized clinical trial. J Clin Oncol. 2004 May 01;22(9):1583-8. PubMed PMID: 15051755.

13. Roa W, Kepka L, Kumar N, Sinaika V, Matiello J, Lomidze D, et al. International Atomic Energy Agency Randomized Phase III Study of Radiation Therapy in Elderly and/or Frail Patients With Newly Diagnosed Glioblastoma Multiforme. J Clin Oncol. 2015 Dec 10;33(35):4145-50. PubMed PMID: 26392096. - A randomized trial comparing hypofractionated radiotherapy to a to a very short course of radiotherapy in elderly glioblastoma patients 
14. Stupp R, Mason WP, van den Bent MJ, Weller M, Fisher B, Taphoorn MJ, et al. Radiotherapy plus concomitant and adjuvant temozolomide for glioblastoma. $\mathrm{N}$ Engl J Med. 2005 Mar 10;352(10):987-96. PubMed PMID: 15758009.

15. Gallego Perez-Larraya J, Ducray F, Chinot O, Catry-Thomas I, Taillandier L, Guillamo JS, et al. Temozolomide in elderly patients with newly diagnosed glioblastoma and poor performance status: an ANOCEF phase II trial. J Clin Oncol. 2011 Aug 01;29(22):3050-5. PubMed PMID: 21709196.

16. Stupp R, Hegi ME, Mason WP, van den Bent MJ, Taphoorn MJ, Janzer RC, et al. Effects of radiotherapy with concomitant and adjuvant temozolomide versus radiotherapy alone on survival in glioblastoma in a randomised phase III study: 5-year analysis of the EORTC-NCIC trial. Lancet Oncol. 2009 May;10(5):459-66. PubMed PMID: 19269895.

17. Perry JR, Laperriere N, O'Callaghan CJ, Brandes AA, Menten J, Phillips C, et al. A phase III randomized controlled trial of short-course radiotherapy with or without concomitant and adjuvant temozolomide in elderly patients with glioblastoma (CCTG CE.6, EORTC 26062-22061, TROG 08.02, NCT00482677). J Clin Oncol. 2016;34:(suppl; abstr LBA2).

-• Randomized trial comparing radiotherapy and temozolomide chemotherapy in elderly patients with newly diagnosed glioblastoma.

18. Nghiemphu PL, Liu W, Lee Y, Than T, Graham C, Lai A, et al. Bevacizumab and chemotherapy for recurrent glioblastoma: a single-institution experience. Neurology. 2009 Apr 07;72(14):1217-22. PubMed PMID: 19349600. PMCID: PMC2677488.

19. Kreisl TN, Kim L, Moore K, Duic P, Royce C, Stroud I, et al. Phase II trial of single-agent bevacizumab followed by bevacizumab plus irinotecan at tumor 
progression in recurrent glioblastoma. J Clin Oncol. 2009 Feb 10;27(5):740-5. PubMed PMID: 19114704. PMCID: PMC2645088.

20. Lai A, Tran A, Nghiemphu PL, Pope WB, Solis OE, Selch M, et al. Phase II study of bevacizumab plus temozolomide during and after radiation therapy for patients with newly diagnosed glioblastoma multiforme. J Clin Oncol. 2011 Jan 10;29(2):142-8. PubMed PMID: 21135282. PMCID: PMC3058273.

21. Chinot OL, Wick W, Mason W, Henriksson R, Saran F, Nishikawa R, et al. Bevacizumab plus radiotherapy-temozolomide for newly diagnosed glioblastoma. $\mathrm{N}$ Engl J Med. 2014 Feb 20;370(8):709-22. PubMed PMID: 24552318.

22. Gilbert MR, Dignam JJ, Armstrong TS, Wefel JS, Blumenthal DT, Vogelbaum MA, et al. A randomized trial of bevacizumab for newly diagnosed glioblastoma. N Engl J Med. 2014 Feb 20;370(8):699-708. PubMed PMID: 24552317.

23. Le Rhun E, Parrott T, Estok T, Gorlia T, Golfinopoulos V, Weller M. EORTC 1608: a phase $1 \mathrm{~b}$ study of TG02, an oral cyclin-dependent kinase 9/5 inhibitor, in elderly patients with newly diagnosed glioblastoma stratified by MGMT promoter methylation status. Neuro Oncol. 2016; 18 (suppl 6): vi8.

24. Weller M, Tabatabai G, Kastner B, Felsberg J, Steinbach JP, Wick A, et al. MGMT Promoter Methylation Is a Strong Prognostic Biomarker for Benefit from Dose-Intensified Temozolomide Rechallenge in Progressive Glioblastoma: The DIRECTOR Trial. Clin Cancer Res. 2015 May 01;21(9):2057-64. PubMed PMID: 25655102.

25. Wick W, Brandes AA, Gorlia T, Bendszus M, Sahm F, Taal W, et al. EORTC 26101 phase III trial exploring the combination of bevacizumab and lomustine in 
patients with first progression of a glioblastoma. . J Clin Oncol 2016;34 (suppl; abstr 2001).

26. Seystahl K, Gramatzki D, Roth P, Weller M. Pharmacotherapies for the treatment of glioblastoma - current evidence and perspectives. Expert Opin Pharmacother. 2016 Jun;17(9):1259-70. PubMed PMID: 27052640.

27. Preusser M, Lim M, Hafler DA, Reardon DA, Sampson JH. Prospects of immune checkpoint modulators in the treatment of glioblastoma. Nat Rev Neurol. 2015 Sep;11(9):504-14. PubMed PMID: 26260659.

28. Weiss T, Weller M, Roth P. Immunotherapy for glioblastoma: concepts and challenges. Curr Opin Neurol. 2015 Dec;28(6):639-46. PubMed PMID: 26402401. 29. Roth P, Preusser M, Weller M. Immunotherapy of Brain Cancer. Oncol Res Treat. 2016;39(6):326-34. PubMed PMID: 27260656.

30. Gilbert MR, Wang M, Aldape KD, Stupp R, Hegi ME, Jaeckle KA, et al. Dose-dense temozolomide for newly diagnosed glioblastoma: a randomized phase III clinical trial. J Clin Oncol. 2013 Nov 10;31(32):4085-91. PubMed PMID: 24101040. PMCID: 3816958. 
Figure legend

Figure 1. Treatment algorithm for elderly patients with newly diagnosed

glioblastoma. MGMT, $\mathrm{O}^{6}$-methylguanine-DNA methyltransferase; RT, radiotherapy; TMZ, temozolomide.

\section{Conflict of interest}

PR has received honoraria for advisory boards and lectures from Roche, MSD, BMS, Novartis and Molecular Partners. DG reports no disclosures. MW has received research grants from Acceleron, Actelion, Bayer, Isarna, MSD, Merck \& Co, Novocure, OGD2, Piqur and Roche and honoraria for lectures or advisory board participation or consulting from BMS, Celldex, Immunocellular Therapeutics, Isarna, Magforce, MSD, Merck \& Co, Northwest Biotherapeutics, Novocure, Pfizer, Roche, Teva and Tocagen. 